\title{
Resting Skeletal Muscle Membrane Potential as an Index of Uremic Toxicity
}

\section{A PROPOSED NEW METHOD TO ASSESS ADEQUACY OF HEMODIALYSIS}

\author{
James R. Cotton, Terry Woodard, Norman W. Carter, and James P. Knochel, \\ Veterans Administration Medical Center, and Department of Internal Medicine, \\ University of Texas Health Science Center, Dallas, Texas 75235
}

\begin{abstract}
A B S T RAC T Electrochemical disturbances of skeletal muscle cells in untreated uremia are characterized by an increase in the intracellular sodium and chloride content, a decrease in intracellular potassium, and a low resting membrane potential. In this study, we have reexamined the foregoing and, in addition, have examined the effects of hemodialysis. Three groups of patients were studied. In the first group of 22 uncomplicated uremic patients, whose creatinine clearance (Ccr) ranged from 2 to $12 \mathrm{~cm}^{3} / \mathrm{min}$ per $1.73 \mathrm{~m}^{2}$, resting transmembrane potential difference $(\mathrm{Em})$ of skeletal muscle cells was measured. In each of the nine patients whose Ccr ranged between 6.3 and $12 \mathrm{~cm}^{3} / \mathrm{min}$, the $\mathrm{Em}$ was normal (i.e., $-90.8 \pm 0.9 \mathrm{mV}$, mean \pm SEM). However, as Cor dropped below $6.3 \mathrm{~cm} / \mathrm{min}$, the Em became progressively reduced and assumed a linear relationship with the Ccr.
\end{abstract}

In the second study, nine individuals with endstage renal disease, whose mean Ccr was $4.3 \mathrm{~cm}^{3} / \mathrm{min}$, underwent measurement of $\mathrm{Em}$ and intracellular electrolyte concentration before and after $7 \mathrm{wk}$ of hemodialysis. Before dialysis, the $\mathrm{Em}$ was $-78.5 \pm 2.1 \mathrm{mV}$, intracellular sodium and chloride were elevated, and the intracellular potassium was reduced. After $7 \mathrm{wk}$ of hemodialysis the Em rose to $-87.8 \pm 1.3 \mathrm{mV}$, and the intracellular sodium, chloride, and potassium became normal.

In the third study, seven patients who were stable on 6-h thrice-weekly dialysis were studied before and after reduction of dialysis to $6 \mathrm{~h}$ twice weekly. In those individuals whose $\mathrm{Em}$ remained normal after $6 \mathrm{wk}$, dialysis time was reduced further. On thrice-weekly

This material was presented in preliminary form at the Annual Meeting of the American Society of Nephrology, Washington, D. C., 1977.

Received for publication 3 March 1.978 and in revised form 6 November 1978. dialysis the $\mathrm{Em}$ was $-91.2 \pm 1.0 \mathrm{mV}$. With reduced dialysis, the Em fell to $-80.1 \pm 0.8 \mathrm{mV}(P<0.001)$. In each case, the Em became abnormal before significant signs or symptoms of uremia were noted. These findings demonstrate that end-stage renal disease is associated with serious electrochemical changes in the muscle cell which are reversed by hemodialysis and recur when dialysis time is reduced. Thus, serial observations of muscle Em may be a potentially powerful tool to assess adequacy of dialysis therapy.

\section{INTRODUCTION}

Previous studies from this laboratory have shown that the resting transmembrane potential difference $(\mathrm{Em})^{1}$ of skeletal muscle cells is abnormally low in patients with end-stage renal disease $(1,2)$. In each instance, the low Em was associated with abnormalities of cellular composition, thus suggesting cellular injury. These include elevated cellular concentrations of sodium and chloride, and reduced potassium. In our experience, these findings occur more consistently than corresponding chemical derangernents of erythrocytes (3) or leukocytes (4) in uremia. Welt et al. could identify this chemical alteration of erythrocyte composition in only $25 \%$ of patients with uremia (3). Thus, the uniform consistency of electrochemical derangements in uremic muscle suggests that this tissue will more reliably mirror impairment of cellular function in uremia.

We assume that at least part of the Em of normal muscle cells results from active sodium transport. Although the reason why the $\mathrm{Em}$ is abnormally low in uremia is not clear, it seems conceivable that defective

\footnotetext{
${ }^{1}$ Abbreviations used in this paper: Cor, creatinine clearance; $\mathrm{Em}$, transmembrane potential difference; $[\mathrm{Na}]^{i}$, intracellular sodium concentration.
} 
sodium transport could be at least partially responsible and, further, that this energy-using process might be suppressed by uremic toxins. If this is the case, adequate removal of the toxins by dialysis should restore the Em to normal, as well as correct the ionic disturbances.

In this report, we have confirmed the previously described electrochemical disturbances of the uremic muscle cell. We now show that hemodialysis can effectively repair these disturbances. It is of interest that once the Em has been corrected, decreasing the frequency of hemodialysis causes a relapse of cellular injury, which suggests that the Em may be a tool to assess adequacy of dialysis therapy.

\section{METHODS}

Patients were selected from the Renal Clinic or dialysis program at the Dallas Veterans Administration Hospital. Those individuals with diabetes mellitus, active infections, malignancy, endocrine derangements, or other concurrent serious illnesses were excluded. The protocol was approved by the Human Studies Committee, and informed written consent was obtained on all study subjects.

29 patients with chronic renal failure were separated into three groups. Group I included 22 patients with chronic renal failure who had various levels of residual renal function. Endogenous creatinine clearance (Ccr) was determined by conventional methods on one or more 24-h urine collections. The resting muscle membrane potential was measured upon completion of the Ccr procedure. All values for Ccr were expressed as $\mathrm{cm}^{3} / \mathrm{min}$ per $1.73 \mathrm{~m}^{2}$ body surface area.

Group II included nine patients from Group I who were studied immediately before chronic dialysis therapy was initiated and again after $7 \mathrm{wk}$ of hemodialysis. Each patient underwent measurement of Em and Ccr. In five of the nine, a sample of skeletal muscle was obtained by needle biopsy from the lateral thigh for quantitation of $\mathrm{Na}, \mathrm{K}$, and $\mathrm{Cl}$ content by methods previously described (2). After $7 \mathrm{wk}$ on standard hemodialysis $\left(6 \mathrm{~h}\right.$ thrice weekly on a $1.0-\mathrm{m}^{2}$ coil or a $1.3-\mathrm{m}^{2}$ hollow fiber dialyzer), the patients were admitted to the Metabolic Unit where the studies were repeated. Em and skeletal muscle electrolyte measurements were made 48-72 h after the previous dialysis.

Group III included seven patients selected from our chronic hemodialysis population. Each had been on dialysis from 6 to 36 mo. They had no abnormalities other than those usually seen in chronically dialyzed patients. In this group, Cor was determined on 48-72 urine collections obtained between two dialyses. Serum creatinine was determined immediately after and before the next dialysis. The average of these two values was used to calculate the Ccr. Throughout this study, six of the patients were dialyzed with a $1.3-\mathrm{m}^{2}$ hollow fiber dialyzer and one with a $1.0-\mathrm{m}^{2}$ coil. Each patient ingested a diet containing $1 \mathrm{~g} / \mathrm{kg}$ of high biological value protein. Their intake was estimated by a research dietitian from a daily food log kept by the patients. The Em was not measured sooner than $66 \mathrm{~h}$ after their last dialysis. The initial measurements were made while patients were maintained on $6 \mathrm{~h}$ thrice-weekly hemodialysis. The dialysis time was then reduced to $6 \mathrm{~h}$ twice weekly. The Em measurements were repeated after $6 \mathrm{wk}$ on reduced dialysis. If the membrane potential remained normal at $6 \mathrm{wk}$, the dialysis time was further reduced in stepwise fashion every $6 \mathrm{wk}$ until the membrane potential became abnormally low.
Resting Em was measured directly in the anterior tibial muscle by the same technique reported previously from this laboratory $(1,2)$, except that for this study we employed a Soltec Rinkidinki chart recorder (Soltec Corp., Sun Valley, Calif.) and a Keithly Electrometer (Keithley Instruments, Inc., Cleveland, Ohio) instead of an oscilloscope. This modification substantially improved the ease and reproducibility with which membrane potential could be determined.

5-15 individual muscle fiber potentials were recorded in each patient. The values reported herein represent an average of all individual fiber potentials from each patient. 23 healthy subjects underwent $\mathrm{Em}$ measurements to serve as normal controls. Their mean Em was $-90.8 \pm 0.9 \mathrm{mV}$ (Mean \pm SEM). The appropriate Student's $t$ test was used for analysis of statistical significance.

\section{RESULTS}

To determine reproducibility of single fiber potentials, data were analyzed from eight patients in whom two electrodes were used during each procedure (Table I). The number of single fiber potentials ranged between 5 and 14 . As compared by the unpaired $t$ test, the average potentials determined from each set of single fiber potentials in any given patient were not significantly different. This reproducibility was evident whether the patients were untreated or treated by hemodialysis. The range of individual muscle fiber potentials during each of these studies is shown in Fig. 1.

Observations from Group I are shown in Fig. 2. Each

TABLE I

Statistical Analysis of Individual Fiber Potentials

\begin{tabular}{|c|c|c|c|c|c|}
\hline & Patient & Electrode & $\begin{array}{l}\text { Number of } \\
\text { potentials }\end{array}$ & $\begin{array}{c}\text { Average } \\
\text { Em }\end{array}$ & SD \\
\hline \multirow[t]{8}{*}{ Uremic } & \multirow[t]{2}{*}{1} & A & 8 & 71.2 & 4.3 \\
\hline & & B & 13 & 73.4 & 8.7 \\
\hline & \multirow[t]{2}{*}{2} & A & 4 & 80.7 & 1.4 \\
\hline & & B & 6 & 78.4 & 2.5 \\
\hline & \multirow[t]{2}{*}{3} & A & 12 & 75.3 & 2.8 \\
\hline & & B & 5 & 75.9 & 0.8 \\
\hline & \multirow[t]{2}{*}{4} & A & 13 & 76.2 & 3.9 \\
\hline & & B & 7 & 76.0 & 6.4 \\
\hline \multirow[t]{8}{*}{ Dialyzed } & \multirow[t]{2}{*}{5} & A & 12 & 92.4 & 5.7 \\
\hline & & B & 11 & 92.4 & 3.1 \\
\hline & \multirow[t]{2}{*}{6} & A & 8 & 99.0 & 8.5 \\
\hline & & B & 9 & 88.6 & 6.6 \\
\hline & \multirow[t]{2}{*}{7} & A & 11 & 83.6 & 3.9 \\
\hline & & B & 7 & 90.0 & 8.5 \\
\hline & \multirow[t]{2}{*}{8} & A & 14 & 91.4 & 4.3 \\
\hline & & B & 13 & 90.8 & 7.6 \\
\hline
\end{tabular}

This table illustrates data derived from individual fiber potentials shown in Fig. 1. The average potentials derived from electrodes $\mathrm{A}$ and $\mathrm{B}$ in any given patient were not significantly different from one another. 


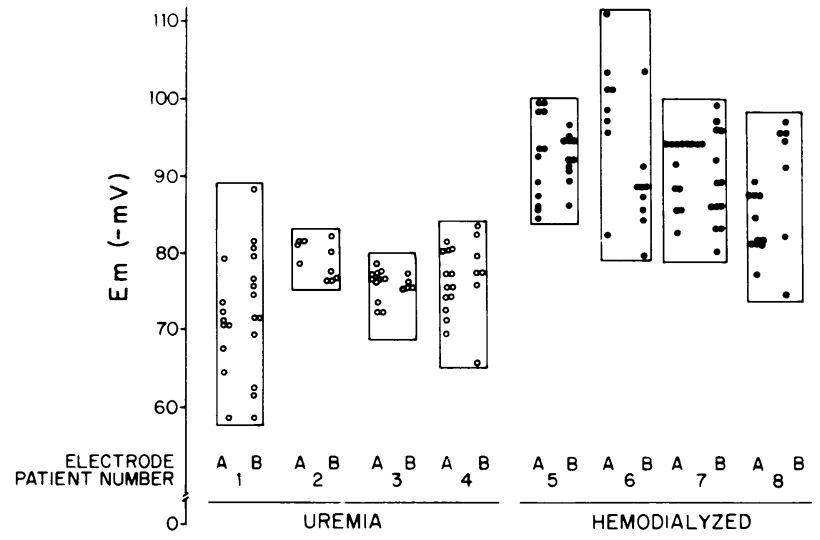

FIgURE 1 Individual muscle fiber potentials obtained by the use of two different electrodes during a given procedure on four untreated (O) patients and four after at least $6 \mathrm{wk}$ of hemodialysis (O). Separate electrodes are designated A and B under their respective values. The electrode values shown in this figure are the source of derived data in Table I.

patient whose Ccr was between 6.3 and $12 \mathrm{~cm}^{3} / \mathrm{min}$ had a normal Em. In contrast, those whose Ccr was $<6.3$ $\mathrm{cm}^{3} / \mathrm{min}$ had an abnormally low Em. In this latter group, the Em bore a linear relationship to the Ccr $(r=0.83 ; \mathrm{Em}=69.4+2.2 \mathrm{Ccr} ; P<0.001)$. Although difficult to express in quantitative terms, there appeared to be a relationship between the presence of uremic symptoms and an abnormally low Em. Eight of the nine patients with a normal Em were asymptomatic. On the other hand, 3 of the 13 with a low Em were clinically uremic. Of the remaining 10,9 complained of some symptoms compatible with end-stage renal disease; primarily those of general malaise and fatigue.

In Group II, the patients had a mean Cor of 4.3 $\mathrm{cm}^{3} / \mathrm{min}$ per $1.73 \mathrm{~m}^{2}$. Eight of the nine patients had

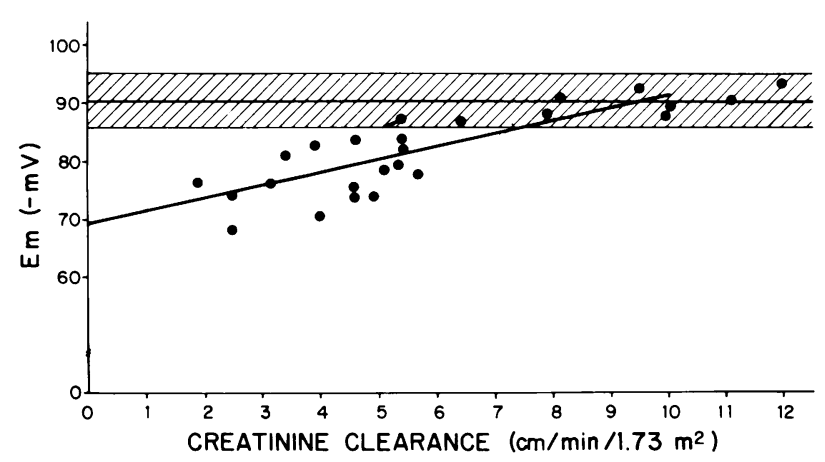

FIGURE 2 Skeletal muscle Em measurements in untreated patients at various stages of chronic renal failure. $25 \mathrm{Em}$ measurements were made in 22 patients, 2 having more than one $\mathrm{Em}$ as their renal function deteriorated. As the Cor fell below $6.3 \mathrm{~cm}^{3} / \mathrm{min}$, the $\mathrm{Em}$ became reduced and assumed a linear relationship to the $\mathrm{Ccr}(r=0.83 ; \mathrm{Em}=69.4 \pm 2.2 \mathrm{Ccr}$; $P<0.001)$. The shaded area represents the mean \pm SD of our normal controls $(-90.8 \pm 4.2 \mathrm{mV})$.
TABLE II

Muscle Composition before and after 7 wh of Dialysis

\begin{tabular}{lccc}
\hline & {$[\mathrm{Na}]^{\mathrm{i}}$} & {$[\mathrm{Cl}]^{i *}$} & {$[\mathrm{~K}]^{i}$} \\
\hline \multicolumn{3}{c}{ meq/liter intracellular $\mathrm{H}_{2} \mathrm{O}$} \\
Normal subjects & $11 \pm 1.4$ & $4.4 \pm 0.1$ & $155 \pm 4.3$ \\
Before dialysis & $26 \pm 4.9$ & $4.8 \pm 0.4$ & $147 \pm 3.1$ \\
After dialysis & $14 \pm 3.1$ & $3.5 \pm 0.5$ & $155 \pm 4.9$ \\
$P_{+}^{+}$ & $<0.01$ & $<0.05$ & $<0.01$ \\
\hline
\end{tabular}

* $[\mathrm{Cl}]^{\mathrm{i}}$, intracellular chloride concentration; $[\mathrm{K}]^{\mathrm{i}}$, intracellular potassium concentration.

$\ddagger$ Statistical analysis by paired Student's $t$ test of values obtained before and after dialysis.

symptoms of advanced renal failure; frank uremia was present in three, and fatigue and malaise in five of the remaining six. Five patients underwent percutaneous muscle biopsy for determination of muscle electrolytes. Data on muscle composition are shown in Table II. These findings were similar to those made previously in end-stage renal disease; i.e., intracellular sodium and chloride were increased, and intracellular potassium was reduced. After $7 \mathrm{wk}$ of hemodialysis, intracellular electrolyte concentrations were normal. Fig. 3 shows the results of the Em measurements before and after $7 \mathrm{wk}$ of hemodialysis. Eight of the nine patients had an abnormal Em before dialysis, and in each, the Em rose in response to hemodialysis. After dialysis, Em in one individual rose, but did not achieve a normal value. All nine patients were asymptomatic at the end of $7 \mathrm{wk}$ of hemodialysis.

The results obtained from Group III patients are shown in Fig. 4. Each of these seven, stable chronic
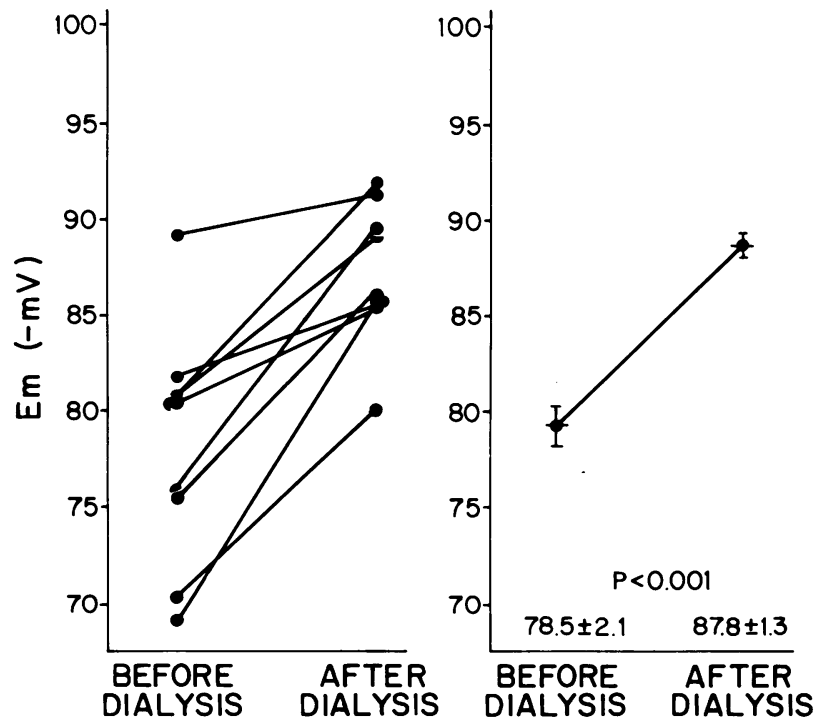

Figure 3 Comparison of the skeletal muscle Em before and after $7 \mathrm{wk}$ of hemodialysis. On the left are individual Em measurements, and on the right are the mean ( \pm SEM) values. 


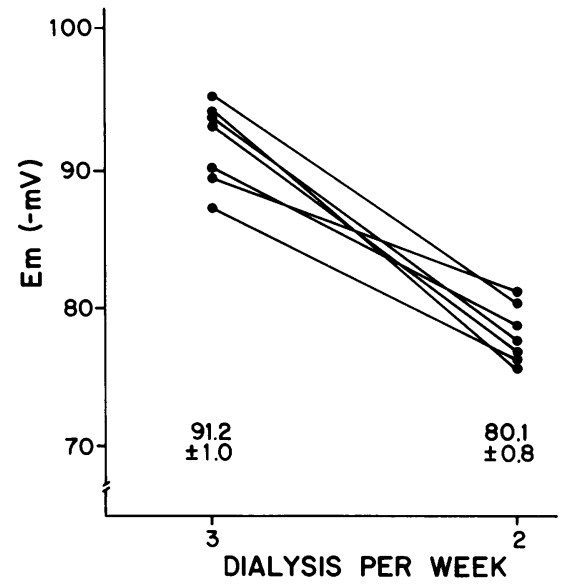

Figure 4 The effect of reduced dialysis on the Em of skeletal muscle. Individual Em measurements obtained on 6-h, thrice-weekly hemodialysis are shown on the left. On the right are the Em values after 6 wk of reduced dialysis $(6 \mathrm{~h}$ or less, twice weekly).

hemodialysis patients had a normal Em on thriceweekly dialysis. After $6 \mathrm{wk}$ of reduced dialysis to 6 $\mathrm{h}$ twice weekly, the Em fell in three patients. Additional reductions in time on dialysis were required in the other four patients. One patient required a reduction to $4.5 \mathrm{~h}$ twice weekly, another to $4 \mathrm{~h}$ twice weekly, another to $3 \mathrm{~h}$ twice weekly, and the last patient to $2.5 \mathrm{~h}$ twice weekly. At the time the Em became abnormal, one patient complained of early morning nausea, and two complained of general malaise. The others remained asymptomatic. Table III illustrates data on body surface area, residual Ccr, and dialysis time per week required to reduce the $\mathrm{Em}$. Minimal dialysis requirements to sustain a normal $\mathrm{Em}$ bore no apparent relationship to either residual renal function or the body surface area.

Table IV depicts laboratory measurements obtained on thrice-weekly dialysis and again on twice-weekly dialysis. Those values shown on twice-weekly dialysis were taken from weekly measurements obtained dur-

TABLE III

Body Surface Area and Ccr in Patients on Reduced Dialysis

\begin{tabular}{cccc}
\hline Patient & Body surface area & Ccr & Dialysis time* $^{*}$ \\
\hline & $m^{2}$ & $\mathrm{~cm}^{3} / m i n$ & $h / w k$ \\
1 & 1.70 & 2.4 & 12 \\
2 & 2.08 & 2.3 & 12 \\
3 & 2.0 & 0.3 & 12 \\
4 & 2.04 & 2.2 & 9 \\
5 & 1.84 & $<0.25$ & 8 \\
6 & 2.0 & 1.15 & 6 \\
7 & 1.70 & $<0.25$ & 5 \\
\hline
\end{tabular}

* Time on dialysis at which $\mathrm{Em}$ returned to low value. ing the 6 wk before developing an abnormal Em. Significant changes were noted in the serum creatinine and hematocrit. No significant change was noted in the blood urea nitrogen, albumin, or potassium.

Fig. 5 demonstrates serial Em measurements in a single patient when dialysis time was varied from thrice to twice weekly on more than one occasion. This man was $53 \mathrm{yr}$ old, was actively working more than $40 \mathrm{~h} / \mathrm{wk}$ in a service station, and stated that he felt well. He had been maintained on twice-weekly dialysis, $6 \mathrm{~h} /$ treatment for $3 \mathrm{yr}$. His Em measurement on this twiceweekly regimen was $-78 \mathrm{mV}$. When dialysis time was increased to $6 \mathrm{~h}$ thrice weekly, his Em measurements initially became abnormally high and returned to normal at $4 \mathrm{wk}$. When dialysis time was decreased to $6 \mathrm{~h}$ twice weekly, the membrane potential returned to abnormally low values. It once again returned to normal after increasing dialysis time to $6 \mathrm{~h}$ thrice weekly. Of interest, the patient subsequently revealed that for the 3 yr while he was on the twice-weekly hemodialysis regimen, he had experienced early morning nausea. On thrice-weekly dialysis this symptom was resolved, and his general well-being improved.

\section{DISCUSSION}

Among the major factors that maintain the electrical potential difference across the plasma membrane of the striated muscle cell are the electrical activity of impermeable intracellular polyanions, differential ionic permeability of the membrane, and sodium transport.

Accordingly, several abnormalities could be responsible for the depressed $\mathrm{Em}$ in uremia. One could be a deficit of high molecular weight intracellular polyanions that normally maintain an electrical diffusion potential for $\mathrm{K}+$. Thus an impermeable anion, such as a protein, could become deficient in concentration, undergo a change in its isoelectric point, or its charges or could become partially neutralized by an uremic toxin. None of these possibilities has been examined.

A second factor responsible for the low Em in uremia could be an increased sarcolemma permeability to sodium ions. If the cellular permeability to $\mathrm{Na}$ ions were increased, and all other factors remained the same, one could predict from the Goldman equation ${ }^{2}$ (2) that the Em would fall. For example, if the normal permeability ratio $(p)$ of $\mathrm{Na}$ to $\mathrm{K}$ is 0.01 , the predicted $\mathrm{Em}$ is $-87 \mathrm{mV}$. An increase of $\mathrm{p}$ to 0.08 would result in an $\mathrm{Em}$ of $-60 \mathrm{mV}$. However, to maintain intracellular concentrations of sodium in the normal range, the

\footnotetext{
${ }^{2} \mathrm{Em}=-61.5 \log [\mathrm{K}]^{\mathrm{i} /}\left([\mathrm{K}]^{\circ}+\mathrm{p}[\mathrm{Na}]^{\circ}\right)$. For this application, assume electrolyte concentrations inside (i) and outside (o) the cell remain unchanged; i.e., $[\mathrm{K}]^{\mathrm{i}}=155 \mathrm{meq} / \mathrm{liter}$; $[\mathrm{K}]^{\circ}=4.5$ $\left.\mathrm{meq} / \mathrm{liter} ;{ }^{\mathrm{Na}}\right]^{\circ}=145 \mathrm{meq} / \mathrm{liter}$. The value for $\mathrm{p}$ is normally 0.01 .
} 
TABLE IV

Laboratory Measurements

\begin{tabular}{lccccc}
\hline & Blood urea nitrogen & Creatinine & Hematocrit & Albumin & $\mathrm{K}^{+}$ \\
\hline & $m g / 100 \mathrm{ml}$ & $m g / 100 \mathrm{ml}$ & vol $\%$ & $\mathrm{~g} / 100 \mathrm{ml}$ & meq/liter \\
Thrice-weekly dialysis & $88 \pm 7.8^{*}$ & $20 \pm 2.5$ & $25 \pm 2.8$ & $4.3 \pm 0.1$ & $4.7 \pm 0.3$ \\
Twice-weekly dialysis $\ddagger$ & $98 \pm 9.8$ & $24 \pm 2.5$ & $22 \pm 2.9$ & $4.2 \pm 0.1$ & $4.8 \pm 0.3$ \\
$P$ & $\mathrm{NS}$ & $<0.01$ & $<0.05$ & $\mathrm{NS}$ & $\mathrm{NS}$ \\
\hline
\end{tabular}

* Mean \pm SEM.

\$ Laboratory values on twice-weekly dialysis are those measured during the 6 wk before developing a low membrane potential.

sodium pump must be capable of responding to this leak in a manner such that sodium ions are extruded as rapidly as they enter the cell. Therefore, to account for our findings, one could propose that either the pump activity itself must be depressed, or its capacity has been exceeded.

Bolte et al. have also reported abnormally low resting membrane potentials in patients with uremia (5). In addition, they examined the effects of acute uremia on electrical properties of fibers from skeletal muscle and from papillary muscles of guinea pig hearts (6). Decreased effective membrane resistance, reflecting increased conductance, and prolonged duration of action potentials were considered to infer increased permeability as a result of primary plasma membrane injury. To our knowledge, no direct studies of cellular permeability have been conducted in either uremic man or animals.

In contrast to the paucity of information on cellular

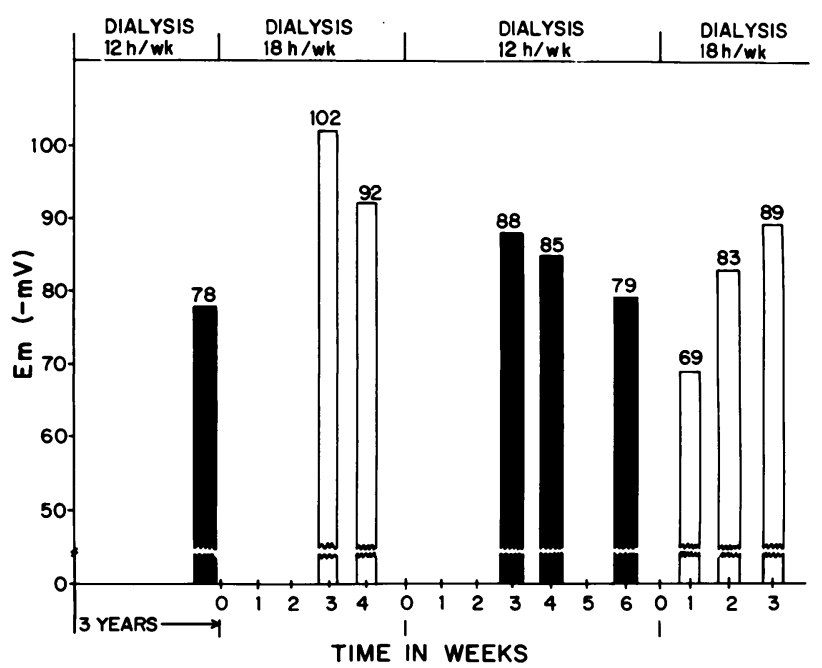

Figure 5 Serial skeletal muscle Em measurements in a single patient. The Em became abnormally low on each occasion when dialysis was reduced to $6 \mathrm{~h}$ twice weekly (12 $\mathrm{h} / \mathrm{wk}$ ), and returned to normal when dialysis time was increased to $6 \mathrm{~h}$ thrice weekly $(18 \mathrm{~h} / \mathrm{wk})$. permeability, numerous investigators have found evidence that active sodium transport is impaired in uremia. The classic study by Welt et al. (3) demonstrated impaired activity of the ouabain-sensitive, Mgdependent, Na-K-activated ATPase, and elevated $\mathrm{Na}$ content of erythrocytes from patients with uremia. These findings have been amply confirmed by others $(7,8)$. Unfortunately, such abnormalities are not found in all patients. Welt et al. found high $\mathrm{Na}$ content and depressed ATPase activity of erythrocytes in $25 \%$ of the patients. Of interest, both defects were more pronounced in those most seriously ill. In contrast to the inconsistent abnormalities in uremic erythrocytes, our observations indicate that the Em bears a linear relationship to endogenous Ccr once the latter falls below $6.3 \mathrm{~cm}^{3} / \mathrm{min}$. Based upon our present and previous studies of seriously ill patients with uremia $(1,2)$, an abnormally low Em has generally been associated with an elevated cellular concentration of $\mathrm{Na}$ (Fig. 6). For this reason, we believe that a depressed Em reflects functional cellular injury. These consistent findings indicate that skeletal muscle is more uniformly affected in uremia than erythrocytes. Although our observations have not identified the specific defect responsible, the evidence would appear to favor either an increase of permeability or suppressed $\mathrm{Na}$ transport, or both.

These studies show for the first time that treatment by hemodialysis can restore both Em and cellular composition to normal. Indeed, serial observations on one case (Fig. 5) whose Em became hyperpolarized after increased dialysis, would be consistent with the notion that the capacity of such an electrogenic transport process may actually be increased, or perhaps even be adapted in chronic renal failure. It seems possible that such an adaptation could occur in response to sustained hyperpermeability of the muscle cell to sodium ions. Even though the capacity for such a transport process could be increased, its activity in the untreated state would remain suppressed by uremic toxins to the extent that the cell could survive; its electrical and compositional characteristics, however, would be altered. If the toxins were removed, the increased capacity of the system 


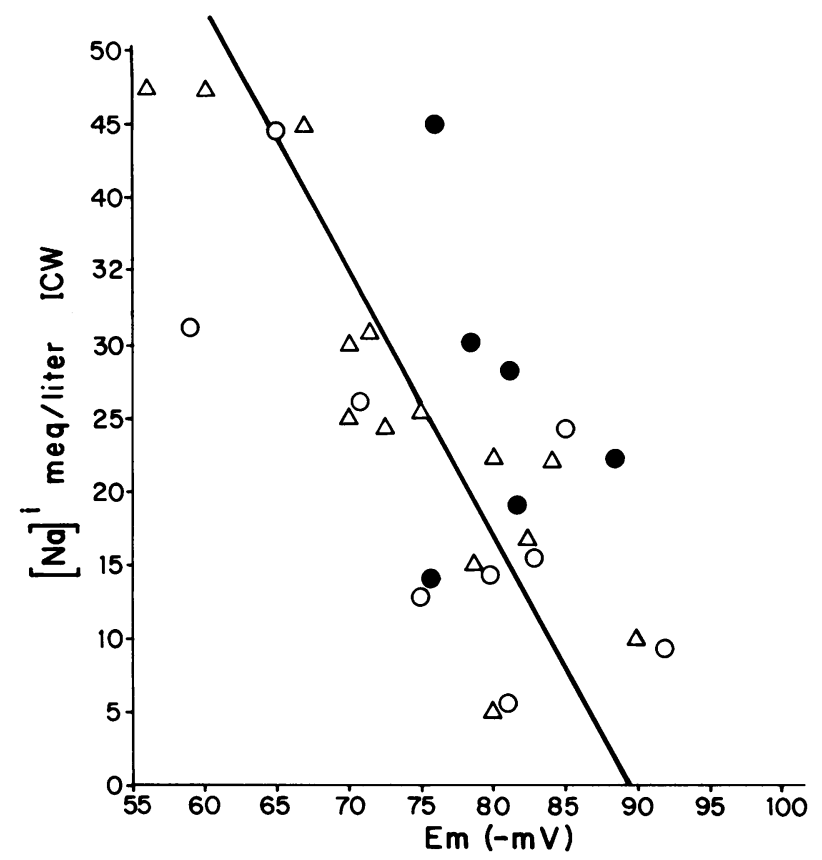

Figure 6 Comparison of skeletal muscle intracellular sodium concentration $[\mathrm{Na}]^{\mathrm{i}}$ and $\mathrm{Em}$ measurements in patients with chronic renal failure. Measurements made previously in our laboratory are depicted by $(\Delta)$ from Cunningham et al. (1) and by (O) from Bilbrey et al. (2). Measurements from this report are shown by the symbol (O). A significant relationship exists between a reduced $\mathrm{Em}$ and an increased $[\mathrm{Na}]^{1}$ in skeletal muscle $\left(r=0.74 ; \mathrm{Em}=89.7-0.6[\mathrm{Na}]^{1}\right.$; $P<0.001)$. ICW, intracellular water.

would be unleashed and hyperpolarization could occur. Hypothetically, such a situation could occur under conditions of increased pump capacity, a continued sodium leak to sustain hyperactivity of a sodium pump, and a lack of suppressive toxins. With continued dialysis therapy, permeability of the cell to sodium would be restored to normal. The latter situation would be consistent with our findings of normal electrochemical characteristics of the cells after 7 wk of adequate dialysis therapy.

We assume that restoration of the electrochemical characteristics of muscle cells to normal by dialysis represents an ideal goal of therapy. Thus it seems possible that serial observations on muscle Em may constitute a potentially powerful tool to assess adequacy of dialysis therapy. Further studies are necessary to evaluate this possibility.

\section{ACKNOWLEDGMENTS}

The authors express their gratitude to Donna Talmon, James Long, Cora Elliott, Julio Borroto, and Patsy Robinson for their contributions to this work.

This study was supported by the Veterans Administration Intramural Research Support fund and U. S. Public Health Service grant 5P01HE11662.

\section{REFERENCES}

1. Cunningham, J. N., N. W. Carter, F. C. Rector, Jr., and D. W. Seldin. 1971. Resting transmembrane potential difference of skeletal muscle in normal subjects and severely ill patients. J. Clin. Invest. 50: 49. (Abstr.)

2. Bilbrey, G. L., N. W. Carter, M. G. White, J. F. Schilling, and J. P. Knochel. 1973. Potassium deficiency in chronic renal failure. Kidney Int. 4: 423. (Abstr.)

3. Welt, L. G., E. K. M. Smith, M. J. Dunn, A. Czerwinski, H. Proctor, C. Cole, J. W. Balfe, and H. J. Gitelman. 1967. Membrane transport defect: The sick cell. Trans. Assoc. Am. Physicians (Philadelphia). 80: 217. (Abstr.)

4. Patrick, J., and N. F. Jones. 1974. Cell sodium, potassium and water in uremia and the effects of regular dialysis as studied in the leucocyte, Clin. Sci. Mol. Med. 46: 583. (Abstr.)

5. Bolte, H. D., G. Riecker, and D. Röhl. 1963. Messungen des Membranpotentials and einzelnen guergestreiften Muskelzellen des Menschen in situ. Klin. Wochenschr. 41: 8. (Abstr.)

6. Bolte, H. D., E. Becker, and W. Völker. 1972. Changes of ionic permeability of skeletal and heart muscle cellular membranes in renal insufficiency. In Uremia: An International Conference on Pathogenesis, Diagnosis and Therapy. R. Kluthe, G. Berlyn, and B. Burton, editors. Georg Thieme Verlag KG., Stuttgart. 14-18.

7. Cole, C. H. 1973. Decreased ouabain-sensitive adenosine triphosphatase activity in the erythrocyte membrane of patients with chronic renal disease. Clin. Sci. Mol. Med. 45: 775. (Abstr.)

8. Kramer, H. J., D. Gospodinov, and F. Krück. 1976. Functional and metabolic studies on red blood cell sodium transport in chronic uremia. Nephron. 16: 344. (Abstr.) 